Methods IF proteins were extracted from individual biopsies in patients with long-standing pan-colitis (LSPC) in clinical, endoscopic and histological remission $(n=10)$ and with recent onset ulcerative colitis (ROUC) also in remission $(n=8)$. Each sample was dot-blotted on a membrane followed by immunoblotting for identification and quantification of keratins (8, 18 and19) sequentially. MCF-7 cell line was used as control in each experiment. Relative Keratins concentration for each dot- blotted sample was inferred by determining its signal intensity relative to the MCF-7 keratins signal intensity measured in turn by densitometry. Statistical analysis to compare the two groups was made separately for K8, K18, K19 using Mann-Whitney U test.

Results Median relative IF protein levels in patients with LSPC were 1.54, 0.41 and 2.12 for K8, K18 and K19, respectively were significantly higher than those with ROUC: $0.03,0.05$ and 0.07 for $\mathrm{K} 8(\mathrm{p}=0.001), \mathrm{K} 18(\mathrm{p}=0.002)$ and $\mathrm{K} 19(\mathrm{p}=0.021)$, respectively. Median Baron's endoscopy score in patients with LSPC and ROUC were 0 (range $0-1$ ) and 1 (range $0-1$ ), respectively. Median histological activity index in both groups were 0 (range $0-1$ ).

Conclusion This study confirms increased expression of insoluble keratins in colonic epithelial cells during LSPC in remission relative to the levels in ROUC and validate our previous MS observations. Restoration of keratins in quiescent LSPC may be a protective mechanism against recurrent inflammation and colorectal cancer.

Disclosure of Interest None Declared.

\section{PTU-065 IS THERE A ROLE FOR FAECAL CALPROTECTIN IN THE INVESTIGATION OF DIARRHOEA IN PATIENTS WITH HIV?}

${ }^{1}$ A Jeevagan*, ${ }^{2}$ LM Clayton, ${ }^{3} \mathrm{~S}$ Soni, ${ }^{4} \mathrm{M}$ Austin. ${ }^{1}$ Gastroenterology, Digestive Diseases Centre, Royal Sussex County Hospital, Brighton, UK; ${ }^{2}$ Academic FY2, Royal Sussex County Hospital, Brighton, UK; ${ }^{3}$ Sexual Health and HIV, Lawson Unit (HIV), Royal Sussex County Hospital, Brighton, UK; ${ }^{4}$ Gastroenterology, Digestive Diseases Centre, Royal Sussex County Hospital, Brighton, UK

\subsection{6/gutjnl-2014-307263.139}

Introduction Diarrhoea is the most common gastrointestinal symptom in patients with HIV. Faecal calprotectin (FCP) is a useful test in the investigation of chronic diarrhoea in the general population. ${ }^{1}$ The sensitivity of this test in HIV-patients with chronic diarrhoea is unknown.

Methods HIV-positive patients undergoing investigation for CD between January 2011 and August 2013 were identified. Demographics and clinical data including measurement of FCP and endoscopy findings were taken from the patients medical records Results 60 patients were referred by the HIV team to Gastroenterology clinic for investigation of CD. There were 55 (92\%) males, mean age was 44 years. All were receiving antiretroviral therapy. No patients had a previous history of Inflammatory Bowel Disease (IBD), 59/60 had negative stool cultures. One patient was diagnosed with giardiasis and excluded from the study. Of the remaining, 54/59 (92\%) patients had FCP measured, of which 36 (67\%) demonstrated inflammation. Of these $31 / 36(87 \%)$ patients with elevated FCP underwent lower GI endoscopy. 9/31 (30\%) patients had abnormal macroscopic findings including mild non-specific inflammatory changes (4/31), polyps (2/31), threadworms $(1 / 31)$ and ileitis (2/31). None had evidence of IBD.

Conclusion In HIV positive individuals receiving antiretroviral therapy 30\% patients with elevated FCP had macroscopic disease. No patients had a diagnosis of IBD. No cause beyond anti-retroviral medication was found. FCP is not a useful test to investigate chronic diarrhoea in this patient cohort.
REFERENCE

1 Konikoff MR, Denson LA. Role of fecal calprotectin as a biomarker of intestinal inflammation in inflammatory bowel disease. Inflamm Bowel Dis. 2006 Jun;12 (6):524-34

Disclosure of Interest None Declared.

\section{PTU-066 A MODEL TO ASSESS THE COST OF FLARE IN ULCERATIVE COLITIS (UC) TO THE NHS}

${ }^{1} \mathrm{~A}$ Bassi*, ${ }^{2} \mathrm{~K}$ Tolley, ${ }^{3} \mathrm{G}$ Wiseman, ${ }^{4} \mathrm{~S}$ Shaw. ${ }^{1}$ Gastroenterology Department, Whiston Hospital, Prescot, Merseyside; ${ }^{2}$ Tolley Health Economics Consultancy Ltd, Buxton; ${ }^{3}$ Medical Affairs, Warner Chilcott (UK) Ltd, Weybridge; ${ }^{4}$ Policy Matters LLP, Surrey, UK

\subsection{6/gutjnl-2014-307263.140}

Introduction Disease flare in ulcerative colitis (UC) can result in substantial cost implications to the NHS. While the costs associated with treatment and management of UC are well-documented, estimates of the cost of flare are lacking. A cost analysis was performed to construct a model to estimate the costs associated with managing a flare across variable pathways.

Methods A decision tree model was developed in Excel to estimate the direct medical costs of flares of various clinical severity. Within the model, the baseline UC patient cohort was maintained on Asa$\mathrm{col}^{\circledR}$ (mesalazine) at a maximum dose of $2.4 \mathrm{~g} /$ day. Simplified care pathways were mapped, assuming that patients would either be treated and managed in primary care, or as an outpatient, or admitted to hospital. Taking a conservative approach, costs for surgery and other procedures e.g., stoma care were excluded as these outcomes are rare and inclusion would significantly increase average flare cost estimates. Treatment and management strategies were based on best practice guidelines, published data sources and clinical expert opinion. Drug costs were calculated using the British National Formulary (BNF) and healthcare management costs were based on published unit costs. The relative proportions of patients likely to follow each route of the treatment pathway were estimated and weightings were applied to enable calculation of an average cost of flare.

Results The estimated annual cost to manage a patient with UC in remission was $£ 955$. The estimated cost to control a flare in primary care was $£ 175$ and for secondary care outpatient management was $£ 578$. For secondary care inpatient management, the estimated cost was $£ 3488$. If a biologic/ciclosporin was needed, the estimated cost rose to $£ 4272$. All costs were inclusive of clinical investigations and treatment reviews. The proportions of patients managed via each pathway were applied to calculations resulting in an estimated average cost of flare of $£ 984$.

Conclusion A model was developed, based on simplified decision tree pathways to enable estimation of the cost of flare in UC. Depending on the severity of the flare episode, costs ranged from $£ 175$ to $£ 4272$. In the future, this model can be used for economic evaluations of interventions to reduce the risk of flare in UC and to help understand the costly aspects of managing ulcerative colitis.

Disclosure of Interest A. Bassi Speaker bureau with: Warner Chilcott (UK) Ltd, K. Tolley Consultant for: Warner Chilcott (UK) Ltd, G. Wiseman Employee of: Warner Chilcott (UK) Ltd, S. Shaw Consultant for: Warner Chilcott (UK) Ltd.

\section{PTU-067 THE FATE OF FLAT LOW-GRADE DYSPLASIA IN ULCERATIVE COLITIS}

${ }^{1}$ A Bond*, ${ }^{2}$ F Campbell, ${ }^{1}$ P Collins. ' Gastroenterology, Royal Liverpool University Hospital, Liverpool, UK; ${ }^{2}$ Histopathology, Royal Liverpool University Hospital, Liverpool, UK

10.1136/gutjnl-2014-307263.141 